\title{
About the existence of conformal metrics on a closed Riemannian manifold $(M, g)$ in a such way that a closed $p$-form $\omega$ is harmonic.
}

Henrique Nogueira de Sá Earp (PQ), Leonardo Francisco Cavenaghi (IC).

\section{Abstract}

We present a sufficient condition for the existence of a conformal metric $h$ on a closed Riemannian manifold $(\mathrm{M}, \mathrm{g})$ with a closed $\mathrm{p}$-form $\omega$ in a such way that $\Delta_{h} \omega=0$.

Key words: Hodge theory, Laplace operator, harmonic forms.

\section{Introduction}

By Hodge theory (1930), for a closed $p$-form $\omega$ be harmonic in a closed Riemannian manifold $(\mathrm{M}, \mathrm{g})$ is necessary and sufficient that $\omega$ be co-closed.

$$
\Delta_{g} \omega=0 \Leftrightarrow d \omega=\delta \omega=0 .
$$

We know, however, that $\delta=*_{g} d *_{g}$ and the condition "be co-closed" depends on the metric. This allows ask:

Given a closed $\mathrm{p}$-form $\omega$ on a closed Riemannian manifold $(M, g)$, when does there exist a metric $h$ on $\mathrm{M}$ in a such way that $\Delta_{h} \omega=0$ ?

This question was answered completely for 1 forms by Calabi in 1969.

We present a sufficient condition for the solution in the case of $p$-forms in each open of some atlas over M.

\section{Results and Discussion}

We search for a metric

$$
h=e^{\frac{2 f}{n}} g
$$

where $f: M \rightarrow \mathbb{R}$ is a $C^{\infty}$ function.

Let $*_{g}$ the Hodge star on the metric $g$. For $\omega$ be co-closed on h-metric:

$$
d *_{h} \omega=0 .
$$

Then the condition to $\omega$ be co-closed is the existence of a function $f \in C^{\infty}(M)$ such that

$$
d f \wedge *_{g} \omega=-d *_{g} \omega
$$

We rewrite it in terms of a matrix equation:

Using the multi-index notation, any $p$-form $\omega$ can be written as:

$$
\begin{gathered}
\omega=\sum_{I} \omega_{I} d x^{I}, \\
I=\left\{i_{1}<\cdots<i_{p}\right\} .
\end{gathered}
$$

Let $J$ be the multi-index complementary of $I$.

Then we define the vector $S^{J}$ of $\mathbb{R}^{n}$ whose coordinates $j=1, \ldots, n$ are:

$$
\left(S^{J}\right)_{j}:=\left\{\begin{array}{cl}
(-1)^{\alpha} \omega_{\left(J-i_{k}\right),} & j=i_{k} \\
0, & j \text { not in J. }
\end{array}\right.
$$

where $\alpha$ means the signal of permutation to rearrange $J$ as an ascending multi-index.

Let A the matrix whose entries are $S^{J}$, and choose a column vector $\mathrm{c}$ whose components are $-\nabla . S^{J}$.

Then we rewrite the PDE (1) as:

$$
\nabla f^{t} \cdot A=c
$$

\section{Conclusions}

Once the problem reduces to linear algebra, the sufficient condition for the existence of conformal metric is given by the invertibility of $A$.

But since $A$ in invertible we get:

$$
\nabla f^{t}=c \cdot A^{-1}
$$

Then the solution $f$ is obtained by integration in each open set of an atlas over $M$.

\section{Acknowledgement}

I would like to thank H. Sá Earp and L. Sperança. This project, range number 2014/06913-0, was financed by FAPESP.

\footnotetext{
Alcibiades Rigas, Sandro B. Cristiano, Francesco Mercuri.
} Differential Forms and their Integrals. 